\title{
Development of a dynamic tool for aircraft noise reproduction
}

\author{
Massimo Viscardi ${ }^{1, *}$ and Maurizio Arena ${ }^{1}$ \\ ${ }^{1}$ Università degli Studi di Napoli “Federico II”, Industrial Engineering Department, Aerospace Section, Via Claudio, 21 - 80125 - \\ Napoli, Italy
}

\begin{abstract}
The aircraft is surely one of the most considerable invention that changed the transport-engineering field, since flying was already an ancient dream come true just a few years ago. Now it is really easy to reach many far places even if most people have no clue how flying is possible. However, as factories do, these large and faster and faster machines return a consistent amount of pollution every day. During take-off, engines reach the highest RPMs returning the most noise possible, and during landing, mobile surfaces produce a lot of aerodynamic disturbs releasing energy in the air while landing gears constantly produce drag in both circumstances. The need to be able to predict the sound emission of an acoustic source represents an extremely current engineering challenge: in particular, a numerical code that would let the user to listen noise produced by a flyover, since acoustic reports are just numerical statistics and spectrogram plots. In this paper, a numerical formulation is suggested for the prediction of the acoustic emission in the frequency domain. The main task of the project was to develop a program that makes dynamic analysis of the signal taking into account the source movement. Moreover, the simulations predicted the noise levels, thus explicitly accounting for the scattering acoustic effects of incidence and geometrical obstacles as well. Geometrical reflections and absorptions of certain frequencies depending on the material have been comprised in the model.
\end{abstract}

\section{Introduction}

The interest toward the environmental impact of transports and noise pollution is reaching growing attention at global level. Among the several objectives set by the ACARE up to 2020, in addition to a drastic reduction of $\mathrm{CO}_{2}$ emissions, the challenge is posed to reduce the noise emitted by 65 percent in order to make EU a centre of excellence and "greening" example, [1]. To achieve these ambitious results is necessary to combine significant advancements in a very varied research fields, enforcing multidisciplinary strategies and transversal objectives. It is clear that these improvements are largely addressed to the aviation sector. Research communities are currently working on issues related to internal noise for the improvement of comfort levels and as mentioned in the emission of emissions in the open environment, [2-12]. Most of the low-altitude noise is emitted just in the vicinity of population centres, increasing the noise level, [13-18]. The problem of optimization of external noise involves the need to provide predictive tools to support all engineering processes. The reconstruction of the sound pressure level is certainly a considerable objective among these. The present work shows some results aimed at predicting the sound pattern in the environment from a point source. Considering the example of a general aviation aircraft, the dynamics equations have been implemented to construct the trajectory of the source in space. The simulation code that has been developed entirely in the Matlab environment, takes into account the relative movement between source and receiver on the basis of the Doppler effect equations.

\section{Tool features}

The main purpose of this activity is to reconstruct the noise trajectory produced by a flyover, since the few acoustic reports are just based numerical statistics and spectrogram plots. So the main task was to develop a program that solved dynamic analysis of the signal because the movement of source. In addition, the model calculated the influence of geometrical obstacles taking into account the surrounding geometrical reflection and materials absorption. The program let the user input many obstacles he wants, so it should be possible to create interesting scenarios, like a plane lifting off surrounded by airport buildings. Since there could be many reflexing obstacles (that consist of flat finite rectangles) the analysis is made on different channels. Every rendered sound concerning a particular obstacle is managed and recorded into a particular channel. When simulation ends the resultant noise is just the sum of what's been 'listened' on these channels, thanks to the superposition principle.

\subsection{Geometric obstacles}

The code can both simulate a scenario and animate the results. To make this happen the program has to save in a matrix the history of position of source in time and of all reflexing points (if they occur) on the obstacles and of course also the track of the rendered noise (or the

* Corresponding author: massimo.viscardi@unina.it 
spectrogram). Such matrix is saved as database into a .mat file, so that only the program can unfold what it features to animate. This obstacles are supposed to be finite rectangles, with wanted orientation and position in space, Fig. 1.

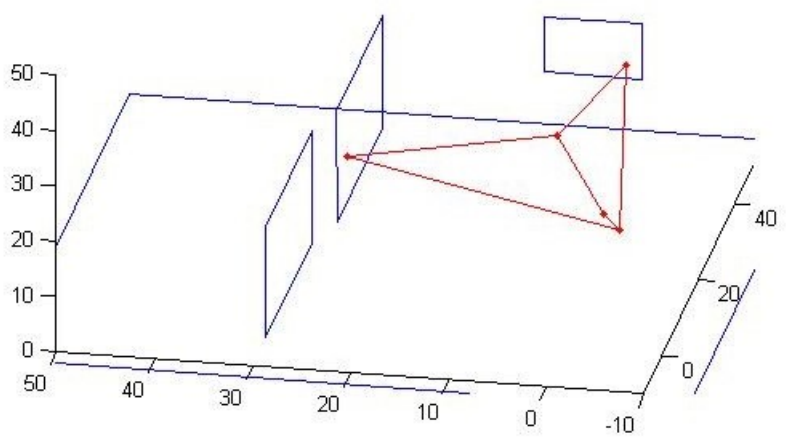

Fig. 1. Introduction of the obstacles in the model.

\subsection{Reflection signal incidence}

In many practical cases, it could happen reflection is not symmetrical; the model calculates where the reflection occurs anyways. Since the processing instructions are featured in a customized routine, once main function calls it than the reflection angle $\Delta \theta$ is asked by the routine itself, Fig. 2.

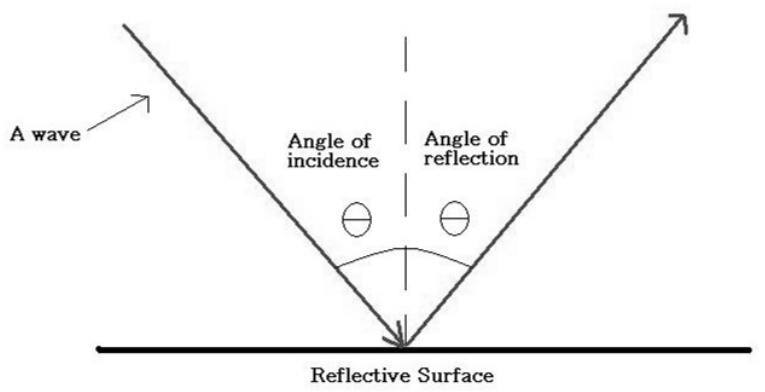

Fig. 2. Definition of reflection angle.

If this value is zero, it means reflection is perfectly symmetrical. This routine tends to be slow at processing, because an analytic solution for the model is not easy to find, so the routine has to calculate it numerically with 3 settable zero-check methods: bisection, chords and secants. The last one is the fastest and the most efficient.

\section{Acoustic source dynamics}

\subsection{Trajectories evolution}

Different kind of trajectories could be implemented in the virtual environment. The dynamics of the sound "disturb" must be therefore modelled. This means that if a sum of force vectors is applied onto a mass point, then its acceleration vector is equal to that sum divided by its inertial mass. Analytically, it poses the resolution of a differential equations system:

$$
m \frac{\partial^{2}}{\partial t^{2}}(x(t), y(t), z(t))=\sum_{i=1}^{N} F(i)
$$

With:

$$
\left\{\begin{array}{c}
x\left(t_{0}\right)=x_{0} \\
y\left(t_{0}\right)=y \\
z\left(t_{0}\right)=z_{0} \\
v_{x}\left(t_{0}\right)=v_{x, 0} \\
v_{y}\left(t_{0}\right)=v_{y, 0} \\
v_{z}\left(t_{0}\right)=v_{z, 0} \\
a\left(t_{0}\right)=a_{x, 0} \\
a_{x}\left(t_{0}\right)=a_{x, 0} \\
a\left(t_{0}\right)=a_{x, 0}
\end{array}\right.
$$

The system cited above constitutes an ODE (Ordinary Differential Equations) problem to numerically integrate. The main purpose deals with the combination of multiple kind of trajectories. Just for instance, during lift off a plane first moves along a line then along a parabolic curve when it rises, roughly. Every time a new trajectory begins, last "information" about positions, velocity and acceleration changes into initial conditions. The "status" should be continuously updated in order to follow the evolution progress. It's possible to solve this system thanks to the Matlab ODE45 (Runge-Kutta) routine, with proper initial conditions in which the mass dots represent numerical singularities (Fig. 3).

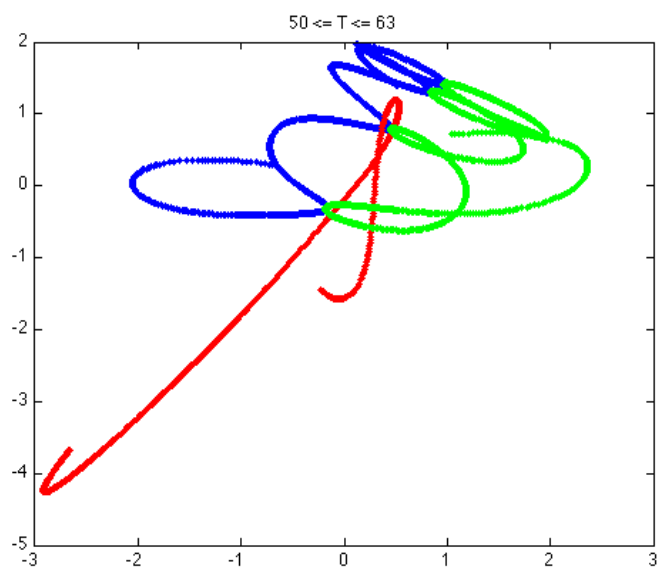

Fig. 3. Multi-path generation.

So potentially many kind of trajectories can be processed, considering the amount of forces applied onto an airplane's gravity centre.

\subsection{Acoustic signal reconstruction}

The Matlab features can make sound a sampled signal if the sampling frequency is assigned as an input data. Every value is separated from the previous one by a time delay, so is the sampling frequency. The value of $44100 \mathrm{~Hz}$ as 
standard frequency with 16bits of resolution per sample have been used for the information stream. If as input a lower frequency is settled, the signal sounds will like slower. To filter the signal, the Discrete Fourier Transform (DTF) is implemented to manipulate the amplitudes of the harmonics. Then the anti-transform (IFFT) to finally listen the result. This is the main process of the project. The whole filtering process is summarized below in Fig. 4.

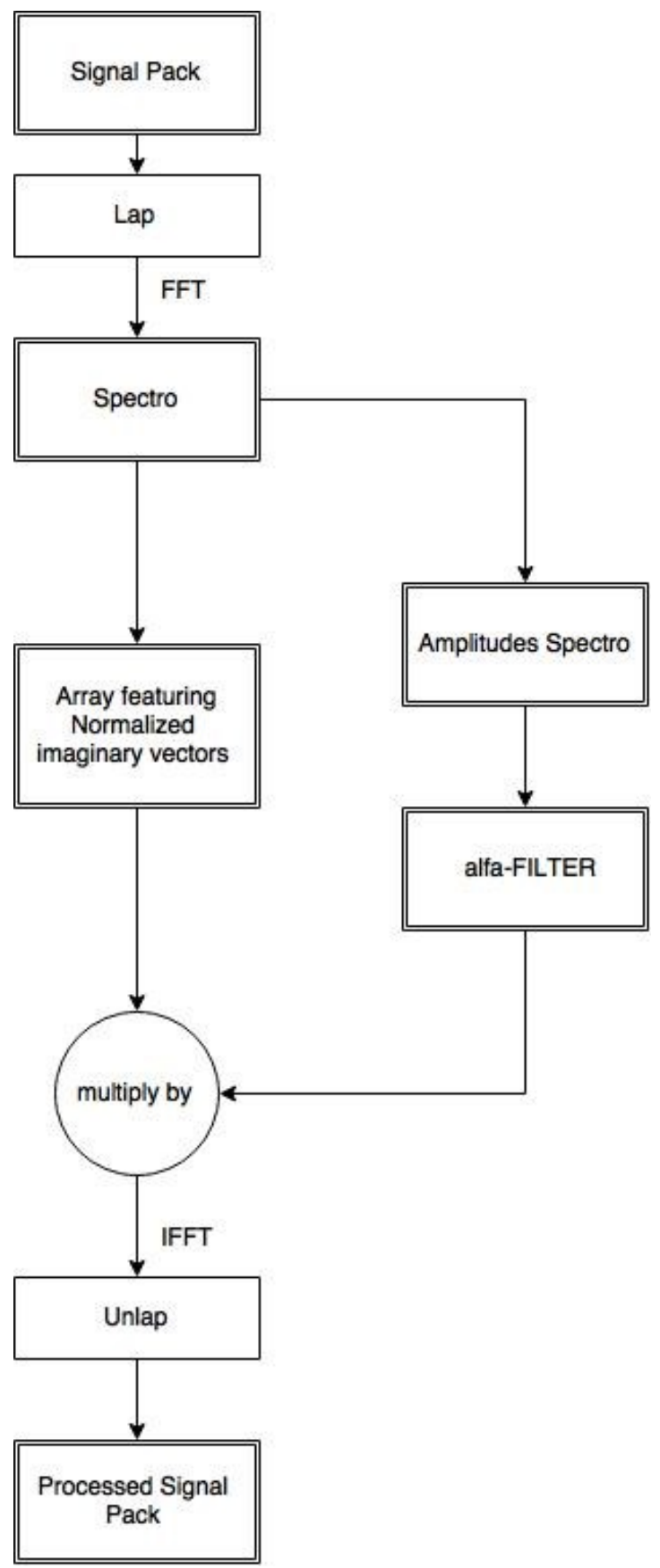

Fig. 4. Filtering process in Matlab.

\section{Results}

Though, to simulate the Doppler effect, packs have not to be too long while on the other hand, they have not to be too short to filter them accurately. Thus, it can be decided that packs' endurance is 0.05 seconds: 2205 samples per pack. In Fig. 5, a plot of given noise by source flying close to the listener fast; it is possible to see the analysis per pack. The source is a $1000 \mathrm{~Hz}$ sinusoid.

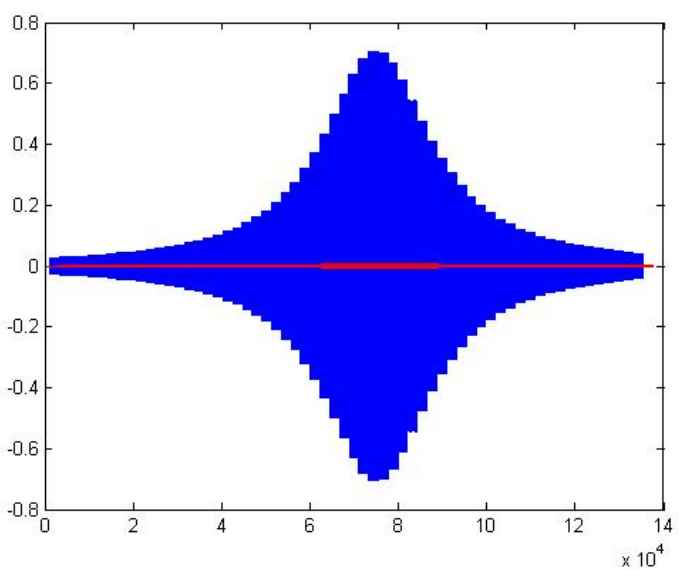

Fig. 5. Noise spectrum of a flying mass dot.

Once the spectrum of a pack is given, it can be filtered it. The alpha-graphic contains information about how material absorbs certain frequencies, Fig. 6. As first test, a sinusoid source flyovers a listener and in Fig. 7 is represented the spectrogram where is clearly evident the shift in frequency due to the doppler effect.

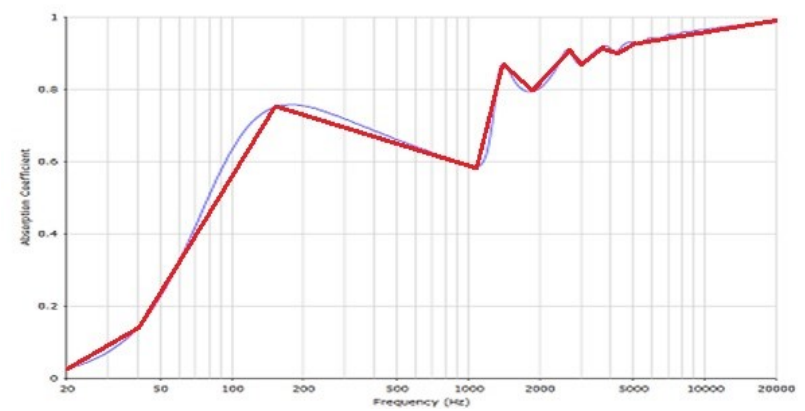

Fig. 6. Absorption coefficient trend.

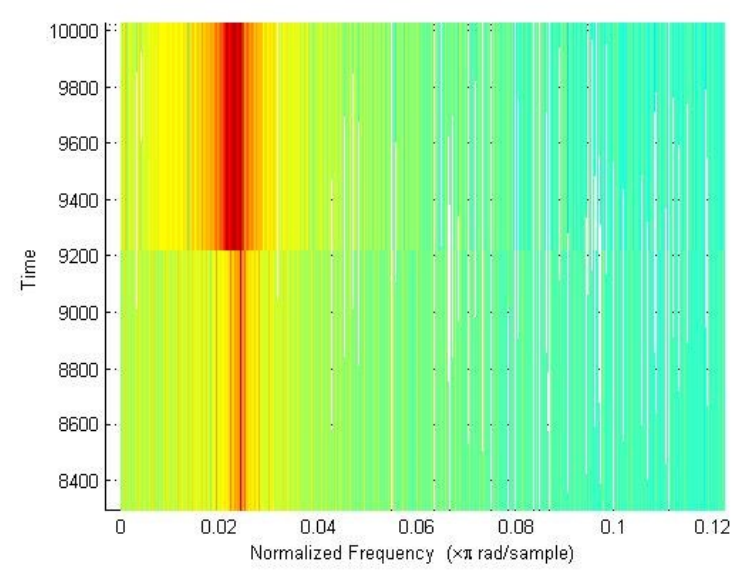

Fig. 7. Spectrogram of the radiating source. 
Other test have been conducted on more classical aircraft noise, as those related to an Airbus A391 Flyover ,Fig. 8.

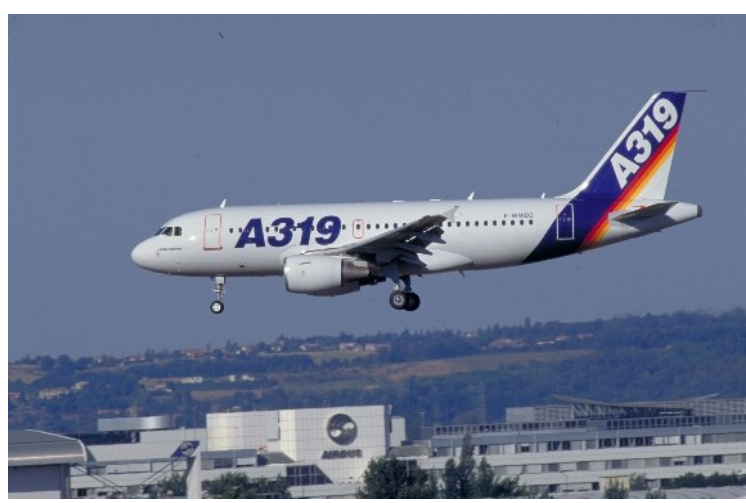

Fig. 8. A319 Flyover noise source

In next Fig. 9 and Fig. 10 the relative time domain and frequency domain noise signature are reports as simulated by the tool.

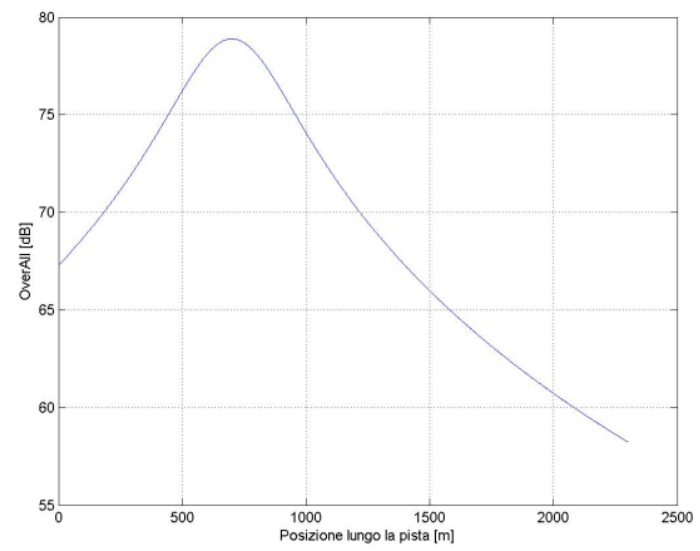

Fig. 9. A319 Flyover noise signature (time domain)

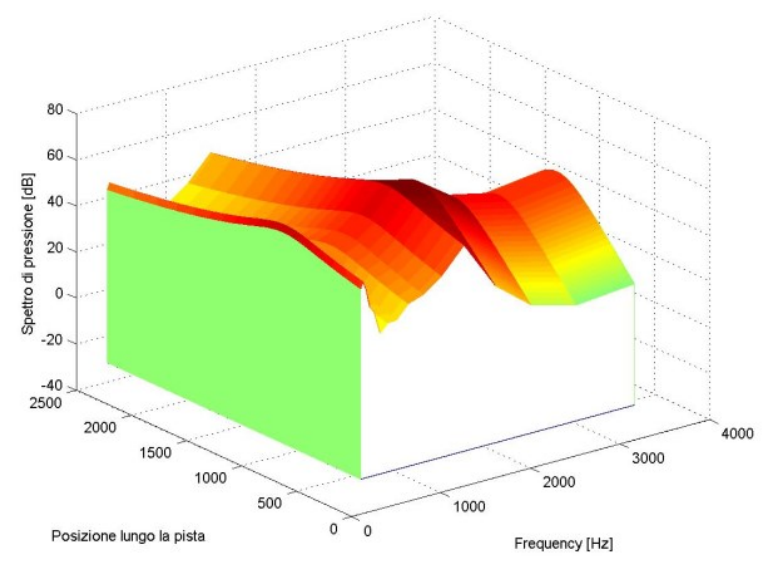

Fig. 10. A319 Flyover noise signature (spectrogram)

\section{Conclusions}

The reduction of external noise emitted by aircraft is a current challenge due to the proximity of many airports to population centers. Noise pollution is in fact the focus of many ambitious worldwide research projects. The present work aims to simulate the level of sound emission released by an aircraft according to a generic trajectory. The development environment was entirely in Matlab. The simulation code solves the differential equations of dynamics over time using a step integration method (Runge-Kutta of the $4^{\text {th }}$ order). The time evolution in spatial dimension is converted by frequency analysis in a sampled signal. The implementation of Doppler effect allowed for consider the approaching and therefore the going away phase of the acoustic source with respect to a fixed receptor in the space. Moreover, the possibility of also inserting any reflecting bodies with the respective absorption coefficients simulates the variation of the sound field. The whole rational process is reported as a workflow in Fig. 11. Future studies will be addressed to manage more complex geometrical bodies as well as the combination of more acoustic disturbances with different pattern of propagation.

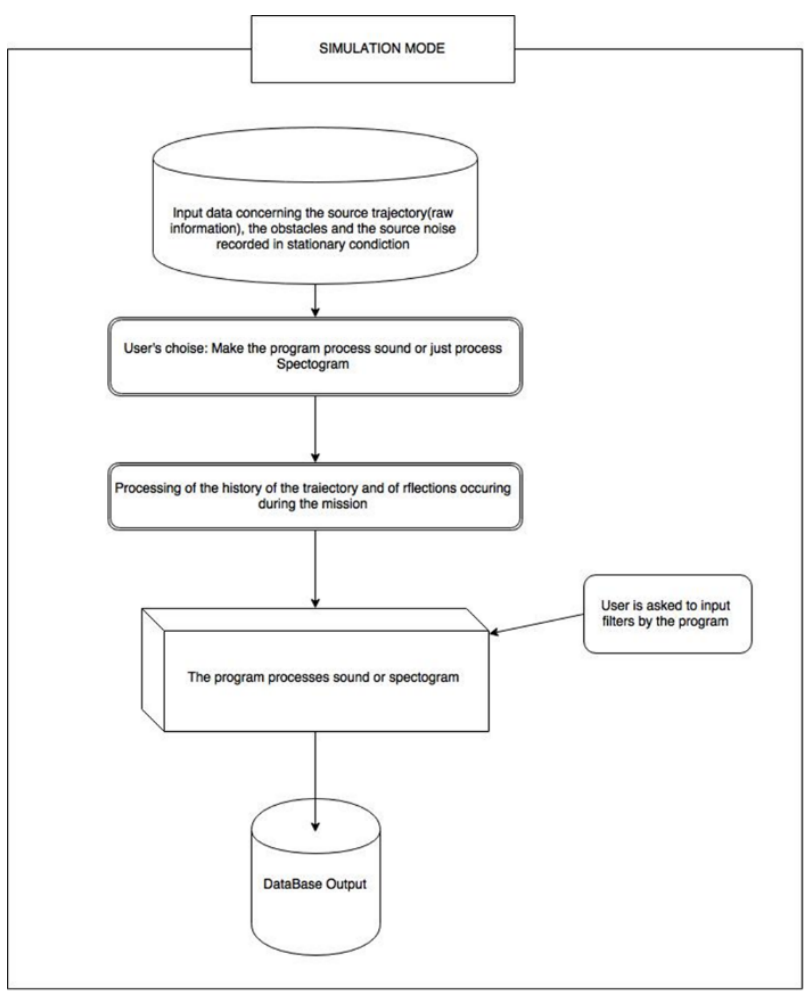

Fig. 11. Summary of the conceptual scheme.

\section{Acknowledgment}

Authors would acknowledge Mr. R. Aprea for his strong contribution to the work during his degree thesis activities. 


\section{References}

1. http://www.acare4europe.com/

2. M. Viscardi, M. Arena, D. Siano, Experimental and numerical assessment of innovative damping foams. International Journal of Mechanics 10, 329-335 (2016).

3. M. Viscardi, M. Arena, D. Siano, Design and testing of a prototype foam for lightweight technological applications. International Journal of Mechanics 10, 383-395 (2016).

4. M. Viscardi, M. Arena, D. Siano, Vibro-acoustic response of a turboprop cabin with innovative sidewall viscoelastic treatment. Proceedings of 24th International Congress on Sound and Vibration, ICVS24, London, UK, (2017).

5. M. Viscardi, P. Napolitano, M. Arena, An innovative numerical approach for railway rolling noise forecast. Proceedings of 24th International Congress on Sound and Vibration, ICVS24, London, UK, (2017).

6. M. Viscardi, T. Coppola, F. Quaranta, E. Rizzuto, D. Siano, On field experimental characterisation of the ship sources of acoustic pollution within a commercial harbour. Proceedings of 24th International Congress on Sound and Vibration, ICVS24, London, UK, (2017).

7. M. Viscardi, P. Napolitano, S. Ferraiuolo, An Innovative Procedure for the Rolling Noise Evaluation, MATEC Web of Conferences, 76, 05013, (2016).

8. M. Viscardi, P. Napolitano, Wheel - Rail interaction based on roughness calculation, WSEAS Transactions on Applied and Theoretical Mechanics 12, 173-180, (2017).

9. M. Arena, A. De Fenza, M. Di Giulio, A. Paonessa, F. Amoroso, Progress in studying passive and active devices for fuselage noise reduction for next generation turboprop, CEAS Aeronautical Journal 8(2), 303-312, (2017).

10. M. Viscardi, M. Arena, Sound proofing and thermal properties of an innovative viscoelastic treatment for the turboprop aircraft fuselage, CEAS Aeronautical Journal, under review, (2018).

11. D. Siano, M. Viscardi, M.A. Panza, Automotive Materials: An Experimental Investigation of an Engine Bay Acoustic Performances, Energy Procedia 101, 598-605, (2016).

12. M. Viscardi, M. Arena, G. Barra, L. Guadagno, Smart carbon-epoxy laminate with high dissipation properties for vibro-acoustic optimization in the turboprop aircraft, International Journal of Mechanics 11, 51-57, (2017).

13. E. Murphy, E.A. King, Environmental Noise Pollution Noise Mapping, Public Health, and Policy, book, (2014).

14. R. Girvin, Aircraft noise-abatement and mitigation strategies, Journal of Air Transport Management 15(1), 14-22, (2009).
15. R. N. Lawton, D. Fujiwar, Living with aircraft noise: Airport proximity, aviation noise and subjective wellbeing in England, Transportation Research Part D: Transport and Environment, 42, 104-118,(2016).

16. E. Ganic, M. Dobrota, O. Babic, Noise abatement measures at airports: Contributing factors and mutual dependence, Applied Acoustics, 112, 32-40, 2016.

17. A. Rodríguez-Díaz. B. Adenso-Díaz, P.L. GonzálezTorre, A review of the impact of noise restrictions at airports, Transportation Research Part D: Transport and Environment, 50, 144-153, (2017).

18. M. Lijesen, W. van der Straaten, J. Dekkers, R. van Elk, J. Blokdijk, How much noise reduction at airports?, Transportation Research Part D: Transport and Environment, 15(1), 51-59, 2010. 